\title{
Intervention Required
}

National Cancer Institute

\section{Source}

National Cancer Institute. Intervention Required. NCI Thesaurus. Code C52668.

A patient condition when action or ministration whose purpose is to improve health or to alter the course of a disease is considered essential and mandatory in order to prevent permanent impairment or damage. 